This is the final peer-reviewed accepted manuscript of:

M. Guermandi, E. F. Scarselli and R. Guerrieri, "A Driving Right Leg Circuit (DgRL) for Improved Common Mode Rejection in Bio-Potential Acquisition Systems" in IEEE Transactions on Biomedical Circuits and Systems, vol. 10, no. 2, pp. 507-517, April 2016

The final published version is available online at:

https://doi.org/10.1109/TBCAS.2015.2446753

Rights / License:

The terms and conditions for the reuse of this version of the manuscript are specified in the publishing policy. For all terms of use and more information see the publisher's website.

This item was downloaded from IRIS Università di Bologna (https://cris.unibo.it/)

When citing, please refer to the published version. 


\title{
A Driving Right Leg Circuit (DgRL) for Improved Common Mode Rejection in Bio-Potential Acquisition Systems
}

\author{
Marco Guermandi, Eleonora Franchi Scarselli, Member, IEEE, and Roberto Guerrieri
}

\begin{abstract}
The paper presents a novel Driving Right Leg (DgRL) circuit designed to mitigate the effect of common mode signals deriving, say, from power line interferences. The DgRL drives the isolated ground of the instrumentation towards a voltage which is fixed with respect to the common mode potential on the subject, therefore minimizing common mode voltage at the input of the front-end. The paper provides an analytical derivation of the common mode rejection performances of DgRL as compared to the usual grounding circuit or Driven Right Leg (DRL) loop. DgRL is integrated in a bio-potential acquisition system to show how it can reduce the common mode signal of more than $70 \mathrm{~dB}$ with respect to standard patient grounding. This value is at least $30 \mathrm{~dB}$ higher than the reduction achievable with DRL, making DgRL suitable for single-ended front-ends, like those based on active electrodes. EEG signal acquisition is performed to show how the system can successfully cancel power line interference without any need for differential acquisition, signal post-processing or filtering.
\end{abstract}

Index Terms-Common mode rejection, common mode rejection ratio (CMRR), driven right leg, electrocardiography, electroencephalography.

\section{INTRODUCTION}

$\mathbf{M}$ ULTICHANNEL acquisition systems for bio-potential signals (e.g., Electroencephalography, EEG and Electrocardiography, ECG) are widely used in both research and clinical practice, with systems ranging from a low channel count (e.g., 12 leads for a standard ECG, 21 electrodes for an EEG 10-20 montage [1]) to hundreds of channels (e.g., high density EEG with 256 channels or more [2]). One of the main issues affecting the quality of the signals extracted is power line interference which can either generate displacement currents in the leads from the electrodes to the acquisition system or generate common mode voltages on the subject which may be converted to differential signals if the Common Mode Rejection (CMR) of the system is insufficient [3]. The first effect can be addressed by reducing the distance between the electrode and the first amplification stage, for example using so-called active electrodes [4]-[7]. These systems integrate an amplification stage directly

Manuscript received November 06, 2014; revised February 19, 2015; accepted June 13, 2015. This work was supported in part by the CREAM project. The CREAM project received funding from the European Union under the Seventh Framework Programme, grant agreement 612022. This paper was recommended by Associate Editor Y. M. Chi.

The authors are with the Advanced Research Center on Electronic Systems (ARCES), University of Bologna, Bologna 40123, Italy (e-mail: marco.guermandi@unibo.it).

Digital Object Identifier 10.1109/TBCAS.2015.2446753 on the electrode with the purpose of providing a very high load impedance to the electrode while driving the cable with a low impedance, making the system more robust to interferences on the leads and to the potential divider effect due to the contact impedance between electrode and skin. This allows one to reduce the need for skin treatment and abrasion, even allowing use of dry-electrodes which do not need conductive gel or paste at the skin-electrode interface [8]. On the other hand, the effect of common mode signal coupling to the patient is generally addressed by using differential acquisition schemes with input stages having very high input impedance and Common Mode Rejection Ratio (CMRR).

Taking ECG and EEG as an example, the International Federation of Clinical Neurophysiology (IFCN) requirements for clinical recording of EEG signals call for a CMRR of $110 \mathrm{~dB}$ or higher [1] while international standard IEC 60601-2-27 [9], [10] requires a CMRR of approximately $90 \mathrm{~dB}$ when considering a complete mismatch of the contact impedance on the different electrodes. These values can be achieved by state-of-the-art hardware based on differential acquisition schemes and passive electrodes, for which intensive skin preparation is required to obtain a very good contact quality. On the other hand, systems based on active electrodes are usually designed for easy mounting with mild-to-none skin preparation. Therefore, single-ended acquisition is usually preferred since differential acquisition calls for additional wires to be routed to every electrode increasing the complexity, weight and power consumption of the system. However, this requires additional circuitry in order to reduce the common mode interference on the patient and thus improve CMRR performances of the system. These range from standard Driven Right Leg (DRL) circuits [3] to Common Mode Feedforward (CMFF) [11] and Common Mode Feedback (CMFB) techniques [6]. DRL circuits suffer from stability issues which limit the effective improvement in CMRR while CMFF and CMFB lead to an increased number of signals to be routed (wires in the case of active electrodes, which can be noise sensitive, especially for CMFF). These solutions present a reduction of the common mode interference which is hardly higher than $40 \mathrm{~dB}$ [6], [11]-[14]. In order to fulfill IFCN requirements, this would require a CMRR of the acquisition stage (without considering common mode reduction techniques) of at least $70 \mathrm{~dB}$. This requires the gain of the amplification stage on the electrode to be limited to a few units [4], [5], otherwise the CMRR is limited to lower values with respect to differential schemes [6]. 
This paper presents a novel scheme for increasing CMR performances by decreasing the effective common mode voltage seen on the patient. It exploits the fact that, for safety reasons, isolated power supply is mandatory in electrical devices directly contacting the patient [9]. In order to fix the patient reference voltage to a value compatible with that of the electronic circuits used in the amplification stages without risking saturation, common instrumentation implements grounding electrodes and DRL loops. By contrast, we propose a Driving Right Leg (DgRL) scheme where the potential which is detected by an electrode on the scalp of the patient drives the isolated ground of the instrumentation which therefore follows the oscillations due to the common mode signal. In this way, even a singleended first stage will see very little common mode at its input, automatically rejecting most of the interference. The levels of CMR which can be obtained are significantly higher than those achievable by standard methods, allowing to obtain the required performances in terms of CMRR even with single-ended frontends. The main advantages are in the reduced complexity of the acquisition systems, especially for those based on active electrodes (no need for CMFB or reference signals to be routed to every electrode) for which it allows the gain of the amplification stage on the electrodes to be substantially higher than one without compromising CMRR performances. This allows to relax noise specifications for the following amplification and analog-to-digital conversion stages, ultimately reducing their complexity, costs and power consumption. Additionally, it reduces sensitivity of CMR to electrode-skin contact quality, reducing the need for skin treatment, which is a time-consuming procedure which requires trained personnel and carries risks of infection [15]. This solution shares some aspects with [16] which was proposed to avoid having an additional electrode in DRL circuits. However, that solution has some disadvantages since it presents positive-feedback whose stability requirements lead to limitations in the quality of common mode rejection. Moreover, it is not compatible with systems based on active electrodes with a gain higher than one.

As a proof of concept, we introduce a 16-channels batterypowered bio-potential acquisition system, implementing DgRL in order to boost the CMRR of the system. For sake of simplicity, the system is based on passive electrodes and standard components, mainly Texas Instruments ADS1298 [17] analog front-end. Section II analytically describes in detail how the DgRL circuit works as compared to standard patient grounding and DRL. Section III introduces the acquisition system. Performances of the system are presented in Section IV, while Section $\mathrm{V}$ draws some conclusions.

\section{DRIVING VERSUS DRIVEN Right LeG}

The scheme in Fig. 1 depicts a typical model designed to evaluate how power line interference propagates to the output in a standard bio-potential acquisition system. The ground electrode (EG) is connected to a fixed voltage with respect to the isolated ground of the instrumentation (eventually to the isolated ground itself as in Fig. 1). Due to the finite contact impedance $Z_{G}$, a residual common mode signal $V_{C M}$ will appear across the impedance $Z_{G}$ itself. A differential amplifier with input impedance $Z_{i}$ is connected to two electrodes (EL1

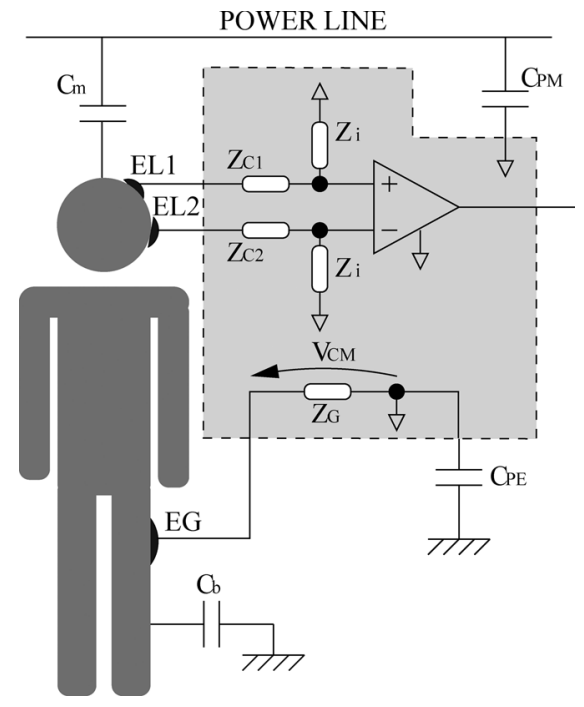

Fig. 1. Simple model for interference coupling from the power line to the subject. $\mathrm{V}_{C M}$ represents the common mode voltage due to parasitic coupling to the mains.

and EL2) for bio-potential signal acquisition, characterized by contact impedances $Z_{C 1}$ and $Z_{C 2}$ respectively. Parasitic capacitances $C_{m}$ and $C_{b}$ model the coupling of the subject to power line and earth respectively, while $C_{P M}$ and $C_{P E}$ model the coupling from the instrumentation isolated ground to power line and earth. This model takes into account only how the interference from the mains translates to a common-mode signal to each electrode. It should be pointed out that, especially when electrodes are far from each other, the interference signal will not be identical on every electrode, therefore leading to a differential contribution which can not be reduced by CMR techniques. This effect becomes more relevant as the number of electrodes increases. Moreover, a higher number of electrodes leads to an increase in parasitic capacitances between instrumentation, body and mains.

We can compute the system CMRR which results from having a differential amplifier with limited CMRR $\left(C M R R_{A}\right)$, a finite input impedance $\left(Z_{i}\right)$ and a mismatch between the contact impedances between skin and the two electrodes $\left(Z_{C 1} \neq Z_{C 2}\right)$. Considering the first issue, the instrumentation amplifiers used in common practice exhibit a very high CMRR (100 dB or more) which is generally large enough to guarantee the required CMR. In the particular case of a system based on active electrodes, single-ended pre-amplification is generally performed on the electrode, which means that every mismatch in the gain of the amplifiers on different electrodes will degrade CMRR [5]. Assuming the two amplifiers have gain $A_{1}$ and $A_{2}$ respectively, $C M R R_{A}$ will be

$$
C M R R_{A}=\frac{A_{1}+A_{2}}{2\left\|A_{1}-A_{2}\right\|} .
$$

It can be proved that due to practical implementation [5], a higher gain leads to a reduction in $C M R R_{A}$, which implies that either the gain is limited to 1 or $C M R R_{A}$ is generally limited to less than $70 \mathrm{~dB}$. In order to increase this value, one possible solution is to calibrate the gain of each amplifier. This requires 


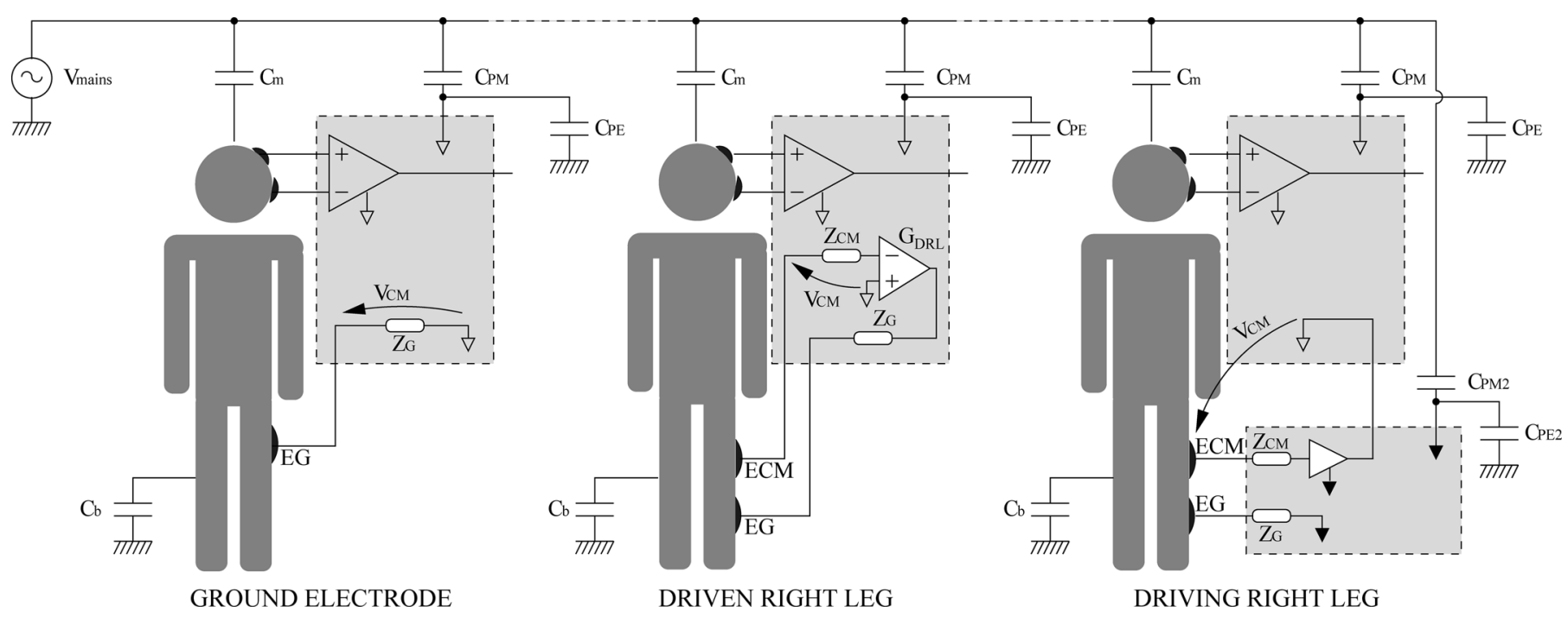

Fig. 2. Comparison between standard patient grounding, DRL and the proposed DgRL.

either performing calibration cyclically in order to compensate for time-dependent variation and/or using very high-accuracy and low-drift components.

In a standard acquisition system (i.e., using passive electrodes and high-CMRR front-ends), the main factor limiting the system CMRR is the voltage divider effect at the differential amplifier input due to the presence of $Z_{i}$ and a mismatch $Z_{C 1} \neq Z_{C 2}$ in the electrode contact impedance [3], which is generally unknown and can vary significantly depending on the quality of the contact with tissue. We can compute a $C M R R_{Z}$ as

$$
C M R R_{Z}=\frac{Z_{i}}{\left\|Z_{C 2}-Z_{C 1}\right\|} .
$$

Equation (2) shows how to maximize $C M R R_{Z}$, the input impedance needs to be as high as possible and the contact impedance needs to be kept as low as possible, eventually requiring substantial skin preparation (i.e., cleaning and abrasion). Assuming that the contact impedance $Z_{C 1}$ can be represented by the parallel of a $51 \mathrm{~K} \Omega$ resistor and a $47 \mathrm{nF}$ capacitance (as required for CMRR measurements in IEC-60601-2-27 [10]), $Z_{C 2}=0$ to take into account the worst case of a complete mismatch and $Z_{i}$ is a $10 \mathrm{pF}$ capacitance, $C M R R_{Z}$ will be equal to $78 \mathrm{~dB}$. For dry-contact electrodes, contact impedance will increase at least of a factor 10 , leading to a reduction of $C M R R_{Z}$ of the same magnitude.

The system $C M R R_{S}$ due to the combination of the two aforementioned effects will be

$$
C M R R_{S}=\frac{1}{\frac{1}{C M R R_{A}}+\frac{1}{C M R R_{Z}}} .
$$

If $C M R R_{A}$ and $C M R R_{Z}$ are not high enough to satisfy specifications, one solution is to use $50 / 60 \mathrm{~Hz}$ notch filters which can however generate distorsions and perform poorly in the case of non-stationary interference. The only additional possibility left to the designer is to implement techniques to reduce the common mode interference $V_{C M}$ before the signals are acquired. If the reduction in the common mode signal is $K_{C M}$, the final CMRR will be

$$
C M R R=K_{C M} \cdot C M R R_{S}
$$

Referring to Fig. 2, we consider three different topologies of patient grounding to reduce $V_{C M}$ in bio-potential acquistion systems. Two electrodes are present in each of them to exemplify signal acquisition. The first scheme presents a standard patient grounding based on one electrode (EG) having the purpose of fixing the patient voltage under the ground electrode as equal to that of the isolated ground. However, parasitic impedances between patient, isolated ground and earth connection lead to a potential divider effect with the electrode contact impedance which restricts the ability to cancel out common mode interference at all accurately. The only way to improve the quality of the common mode interference cancellation is to decrease the contact impedance.

The second scheme in Fig. 2 is a DRL circuit, which is based on negative feedback evaluating the common mode potential on one or more electrodes (ECM) and providing a correction signal on an auxiliary electrode EG so as to minimize the common mode signal itself [3]. Common-mode sensing electrodes can be the same used for signal acquisition. The reduction of common mode interference (and therefore the increase in CMRR) is proportional to the open-loop gain of the DRL itself (approximately equal to $G_{D R L}$ ). Unfortunately, open-loop gain is generally no more than 10 to 100 in order to avoid the risks of instability since the load is not exactly known, being determined by the combination of electrical properties for biological tissues, electrode-skin contact impedances and parasitic elements [6], [12], [18], [19].

The third scheme in Fig. 2 is the proposed DgRL circuit which, instead of attempting to fix the patient common mode to the desired value, uses the potential on the auxiliary electrode ECM to drive the isolated ground of the acquisition system through a high input impedance amplifier that has a nominal 


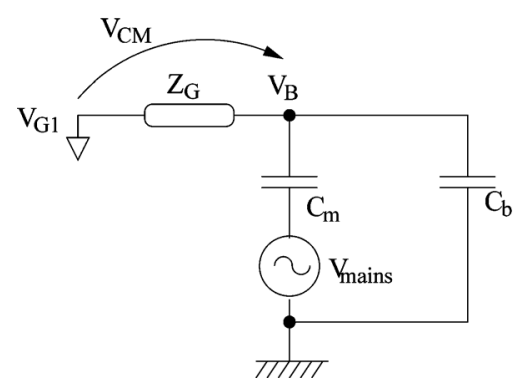

(a)

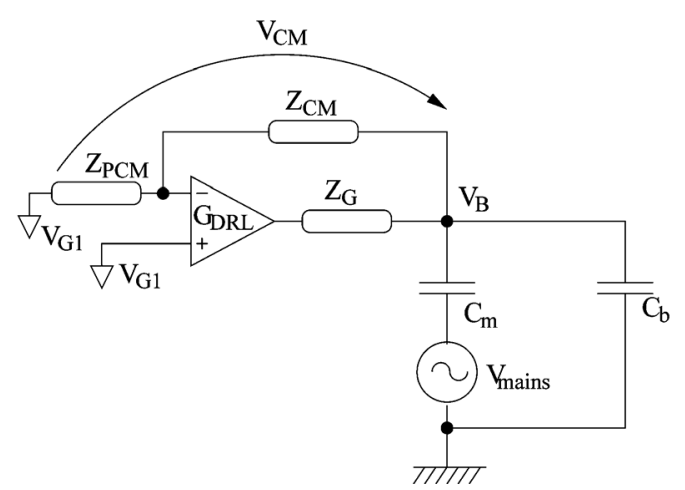

(b)

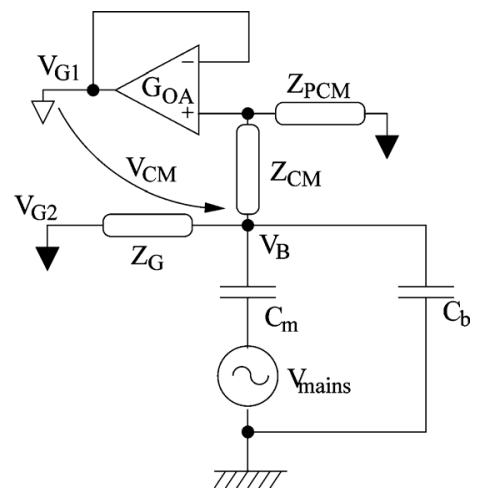

(c)

Fig. 3. Electrical models of standard patient grounding. (a) DRL. (b) The proposed DgRL. (c) $V_{G 1}=\alpha_{1} \cdot V_{\text {mains }}$ and $V_{G 2}=\alpha_{2} \cdot V_{m a i n s}$.

gain equal to 1 . In this way (and unlike what happens with standard patient grounding), since the electrode is connected to a high-impedance stage, the voltage drop across $Z_{C M}$ is significantly reduced with respect to that on $Z_{G}$, minimizing the common mode voltage $V_{C M}$. It should be pointed out that signals from different electrodes might be used to estimate the common mode voltage, for example using a Wilson's central terminal in ECG or earlobe average in EEG.

Fig. 3 presents the electrical models used to evaluate common mode voltage interference on the patient's body due to coupling from the mains in the three cases of standard patient grounding, DRL and DgRL as presented in Fig. 2. $V_{\text {mains }}$ represents the source of the interference (e.g., $220 V_{R M S}$ sine wave at $50 \mathrm{~Hz}$ in Europe, $110 V_{R M S}$ sine wave at $60 \mathrm{~Hz}$ in U.S.), $C_{m}$ is the capacitance coupling the mains to the patient body (usually in the $\mathrm{pF}$ range), $C_{b}$ is the capacitance from the body to earth ground (hundreds of $\mathrm{pF}$ ), $Z_{G}$ is the contact impedance between the grounding electrode and the patient's body, which also takes into account protection resistors included to limit patient auxiliary and fault currents. $Z_{C M}$ represents a similar contact impedance for the electrode detecting the patient common mode in the DRL and DgRL circuit. $V_{B}$ is the potential on the body resulting from mains' interference. Isolated ground potentials $V_{G 1}$ and $V_{G 2}$ are coupled to the mains and ground earth by parasitic capacitances $C_{P E}\left(C_{P E 2}\right)$ and $C_{P M}\left(C_{P M 2}\right)$ as in Fig. 2 . In order to simplify the following derivation, instead of considering explicitly the parasitic capacitances on the isolated ground nodes, we model their effect through two parameters $\alpha_{1}$ and $\alpha_{2}$ which represent the fraction of $V_{\text {mains }}$ which will be present on those nodes $\left(V_{G 1}=\alpha_{1} \cdot V_{\text {mains }}, V_{G 2}=\alpha_{2} \cdot V_{\text {mains }}\right)$. Input impedance of the active circuits and parasitic capacitances affects the quality of common mode reduction, so they are taken into account as an equivalent impedance $Z_{P C M}$ towards isolated ground $\mathrm{G} 1$ or G2. Current flowing on $Z_{P C M}$ is expected to be negligible with respect to that flowing on the other parasitic impedances, while the potential divider effect occuring at the input of the active device is modeled by a parameter $\beta$. Common mode voltage $V_{C M}$ resulting from mains interference is defined as the difference between potentials on the patient's body $V_{B}$ and the instrumentation isolated ground $V_{G 1}\left(V_{C M}=V_{B}-V_{G 1}\right)$.
The common mode voltage can be computed in the three cases to be

$$
\begin{aligned}
V_{C M}^{\prime}(s)= & V_{\text {mains }} \cdot\left[\frac{s C_{m}}{\frac{1}{Z_{G}}+s C_{m}+s C_{b}}+\right. \\
& \left.-\alpha_{1}^{\prime} \cdot \frac{s C_{m}+s C_{b}}{\frac{1}{Z_{G}}+s C_{m}+s C_{b}}\right] \\
V_{C M}^{\prime \prime}(s)= & V_{\text {mains }} \cdot\left[\frac{s C_{m}}{\frac{\left(G_{D R L} \beta+1\right)}{Z_{G}}+s C_{m}+s C_{b}}+\right. \\
& \left.-\alpha_{1}^{\prime \prime} \cdot \frac{s C_{m}+s C_{b}}{\frac{\left(G_{D R L} \beta+1\right)}{Z_{G}}+s C_{m}+s C_{b}}\right] \\
V_{C M}^{\prime \prime \prime}(s)= & V_{m a i n s} \cdot\left[\frac{s C_{m} \cdot\left(1+G_{O A} \cdot(1-\beta)\right)}{\frac{1}{Z_{G}}+s C_{m}+s C_{b}}+\right. \\
& +G_{O A}^{\prime \prime \prime} \cdot \frac{\frac{1}{Z_{G}}}{\frac{1}{Z_{G}}+s C_{m}+s C_{b}}+ \\
& \left.-\alpha_{2}^{\prime \prime \prime} \cdot \frac{\left(s C_{m}+s C_{b}\right) \cdot(1-\beta) \cdot G_{O A}}{\frac{1}{Z_{G}}+s C_{m}+s C_{b}}\right]
\end{aligned}
$$

where $G_{D R L}$ is the DRL amplifier's gain, while $G_{O A}$ is the open-loop gain of the operational amplifier used as a buffer in the DgRL circuit. Parameters $\alpha_{1}^{\prime}, \alpha_{1}^{\prime \prime}, \alpha_{1}^{\prime \prime \prime}, \alpha_{2}^{\prime \prime \prime}$ and $\beta$ can be computed to be

$$
\begin{aligned}
& \alpha_{1}^{\prime}=\frac{Y_{G}\left(s C_{m}+s C_{P M}\right)+s C_{P M}\left(s C_{b}+s C_{m}\right)}{\left(s C_{P E}+s C_{P M}\right)\left(Y_{G}+s C_{b}+s C_{m}\right)} \\
& +Y_{G} \cdot\left(s C_{b}+s C_{m}\right) \\
& { }_{s} C_{m} Y_{C} \\
& \alpha_{1}^{\prime \prime}=\frac{+s C_{P M} \cdot\left(Y_{G}+\beta G_{D R L} Y_{G}+Y_{C}+s C_{b}+s C_{m}\right)}{s\left(C_{P E}+C_{P M}\right)\left[Y_{G}\left(1+\beta G_{D R L}\right)+Y_{C}\right.} \\
& \left.+s\left(C_{b}+C_{m}\right)\right]+Y_{C}\left[Y_{G}+s\left(C_{b}+C_{m}\right)\right] \\
& \alpha_{1}^{\prime \prime \prime} \approx 0 \\
& \alpha_{2}^{\prime \prime \prime}=\frac{s C_{P M 2} \cdot\left(s C_{m}+s C_{b}+Y_{G G}\right)+s C_{m} \cdot Y_{G}}{\left(s C_{P M 2}+s C_{P E 2}+Y_{G G}\right)\left(s C_{m}+s C_{b}+Y_{G G}\right)-Y_{G G}^{2}} \\
& \beta=\frac{Z_{P C M}}{\left(Z_{P C M}+Z_{C M}\right)}
\end{aligned}
$$


where $Y_{G}=1 / Z_{G}, Y_{C}=1 /\left(Z_{P C M}+Z_{C M}\right)$ and $Y_{G G}=$ $\left(Z_{C M}+Z_{P C M}+Z_{G}\right) /\left(Z_{G} \cdot\left(Z_{C M}+Z_{P C M}\right)\right)$.

Since $1 / Z_{G}$ is usually at least one order of magnitude higher than $s C_{b}$ and $s C_{m}$ for $s=2 \cdot \pi \cdot 50 \mathrm{rad} / \mathrm{sec}$ at $50 \mathrm{~Hz}$, the three equations can be approximated as

$$
\begin{aligned}
V_{C M}^{\prime}(s) \approx & V_{\text {mains }}\left[s C_{m} Z_{G}-\alpha_{1}^{\prime} Z_{G} \cdot\left(s C_{m}+s C_{b}\right)\right] \\
V_{C M}^{\prime \prime}(s) \approx & \frac{V_{\text {mains }}}{\left(G_{D R L} \beta+1\right)} \cdot\left[s C_{m} Z_{G}-\alpha_{1}^{\prime \prime} Z_{G} \cdot\left(s C_{m}+s C_{b}\right)\right] \\
V_{C M}^{\prime \prime \prime}(s) \approx & \frac{V_{\text {mains }}}{1+G_{O A}}\left[s C_{m} Z_{G} \cdot\left(1+G_{O A}(1-\beta)\right)+\right. \\
& \left.+\alpha_{2}^{\prime \prime \prime}-\alpha_{2}^{\prime \prime \prime} Z_{G} \cdot\left(s C_{m}+s C_{b}\right) \cdot(1-\beta) G_{O A}\right] .(7)
\end{aligned}
$$

From (7), one can observe how DRL effectively reduces the common mode interference by a factor $K_{C M, D R L}=$ $G_{D R L} \beta+1$. As anticipated in Section I, the need to guarantee stability of the feedback loop limits $G_{D R L}$, and the enhancement in CMR, to approximately $40 \mathrm{~dB}$.

If we now consider the common mode signal in the presence of DgRL ( $\left.V_{C M}^{\prime \prime \prime}\right), G_{O A}$ can easily be as high as $100 \mathrm{~dB}$ without raising stability concerns due to the absence of any high gain feedback loop. Thus we can reduce the third equation of (7) to

$$
\begin{aligned}
V_{C M}^{\prime \prime \prime}(s) \approx V_{\text {mains }} \cdot(1-\beta) \cdot & {\left[s C_{m} \cdot Z_{G}\right.} \\
& \left.-\alpha_{2}^{\prime \prime \prime} \cdot\left(s C_{m}+s C_{b}\right) \cdot Z_{G}\right] .
\end{aligned}
$$

DgRL reduces $V_{C M}^{\prime}(s)$ by a factor $K_{C M, D g R L}=(1-\beta)$ where $\beta$ basically represents the accuracy with which the system can reproduce the common mode signal $V_{B}$ at the input of the unity-gain buffer. Assuming that the contact impedance $Z_{C M}$ can be represented by the parallel of a $51 \mathrm{~K} \Omega$ resistor and a $47 \mathrm{nF}$ capacitance (as required for CMRR measurements in IEC-60601-2-27 [10]) and that $Z_{P C M}$ is a $10 \mathrm{pF}$ capacitance, at $50 \mathrm{~Hz}$ the achievable reduction $\left(K_{C M}\right.$ in (4)) is equal to $78 \mathrm{~dB}$, a value significantly higher than the one which is achievable with a standard DRL circuit. In case dry-electrodes were used, we can assume that $Z_{C M}$ would increase at least by a factor 10 [8], resulting in a CMR in the order of 50 to $60 \mathrm{~dB}$, which can still be enough if the CMRR of the system without the DgRL $\left(C M R R_{S}\right.$ in (4)) is higher than $50 \mathrm{~dB}$. These values can be achieved by dry-electrode systems, provided that active electrode topologies are used [6].

\section{16-Channel EEG Acquisition System With DgRL}

Fig. 4 represents the 16-channel acquisition system for EEG signals used to test performances of the Driving Right Leg circuit. Amplification and analog-to-digital conversion are performed by means of two Texas Instruments ADS1298 analog front-end ICs [17]. Daisy-chain connection allows one to recover data through the Serial Peripheral Interface (SPI) of one of the two devices. A digital optical isolator (Analog Devices ADUM2401 [20]) provides the appropriate level of isolation, as required by IEC-60601 [9] and collateral standards. SPI communication is controlled by Future Devices FT2232H IC, integrated in its mini-module [21]. The device takes care of data exchange with the analog front-end through

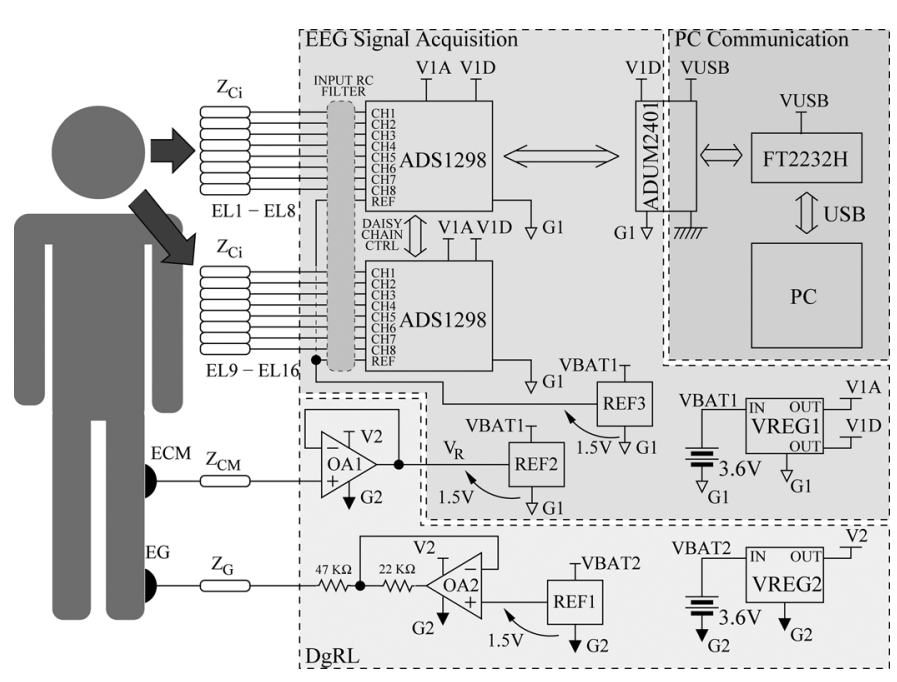

Fig. 4. 16-channel EEG acquisition system with DgRL.

SPI and with the PC for data visualization and recording via a USB connection, both for acquired data and for the front-end program words. Power supply for these blocks is provided by voltage regulators connected to a first battery pack (series of 3 rechargeable NiMH AA batteries, $1.2 \mathrm{~V} 2450 \mathrm{mAh}$ ), except for the FT2232H and the corresponding port of the opto-isolator, which are powered via USB (non-isolated power supply from the mains). A second isolated battery pack is used for the DgRL section.

As discussed in the previous section, the DgRL circuit has the purpose of driving the potential on the isolated ground of the acquisition system G1 towards a voltage which is fixed with respect to the common mode potential on the subject, as detected by electrode ECM. The DgRL drives electrode EG with a potential which, as discussed in the previous section and depending on the contact impedance value $Z_{G}$, is as close as possible to a fixed reference value with respect to isolated ground G2. Amplifier OA1 is used as a unity gain buffer for the signal acquired on the common mode electrode ECM. With respect to the simplified scheme in Fig. 2, the buffer does not directly drive isolated ground G1 but it is connected to a fixed voltage with respect to ground G1. The potential on isolated ground G1 is therefore tied to the potential on node $V_{R}$ and, consequently, will follow the common mode potential detected by electrode ECM. It should be pointed out that, since this is the only low-impedance connection between the acquisition section and the DgRL section of the system, only a very small current will flow on the connection itself. An additional voltage reference provides a voltage of $(\mathrm{V} 1 \mathrm{~A}+\mathrm{VG} 1) / 2$ for the reference input (REF) of the two ADS1298 ICs, which is therefore at a fixed voltage with respect to VG1. This additional voltage reference is not strictly necessary (i.e., $V_{R}$ can be connected directly to $V_{R E F}$ ) and it is inserted only for sake of completeness and clearness in order to show that the acquisition is effectively performed as single-ended (electrode signal versus a potential fixed with respect to isolated ground G1). This shows how, in case of active electrodes where the front-end is placed directly on the electrode, there is no need to send the reference signal to 
every electrode of the system. If bipolar power supply is provided to the ADS1298 ICs, $V_{R}$ can be connected directly to G1 and REF2 and REF3 removed altogether.

\section{A. DgRL Implementation}

The Driving Right Leg circuit basically contains only a voltage reference and the two operational amplifiers OA1 and OA2 configured to act as voltage buffers. OA2 drives the ground electrode EG towards the fixed potential with respect to the isolated ground $\mathrm{G} 2$; two protection resistors are used, for a total value of $69 \mathrm{~K} \Omega$. If a single fault occurs on one of the high-impedance electrodes (EL1 to EL16 and ECM), e.g., a direct connection to the positive power supply or isolated ground, the current flowing through the subject is limited to less than $50 \mu \mathrm{A}$. This is true even if electrode ECM is accidentally detached from the subject and the output of OA1 is free to swing from negative to positive rail. As already discussed, the series of the $47 \mathrm{~K} \Omega$ protection resistor and the contact impedance leads to a potential divider effect limiting the accuracy of the patient grounding and the cancellation of the common mode interference. The potential on the common mode electrode ECM will therefore contain a residual interference from the mains (with respect to isolated ground G2) and it is buffered by means of operational amplifier OA1 which has the function of maximizing the impedance loading the electrode and minimizing the impedance which drives the output of the voltage reference REF2. Since REF2 fixes the potential difference $\left(V_{R}-V_{G 1}\right)$ to $1.5 \mathrm{~V}, V_{G 1}$ will follow the potential on $\mathrm{ECM}$, together with all fixed voltages (e.g., supplies $V 1 A$ and $V 1 D$ and reference input of the front-ends). While characteristics of OA2 are of minor importance in the overall performance of the system, it is important that OA1 is an operational amplifier with high input impedance and low input bias current, so as to minimize the load on the ECM electrode and reduce the possibility of electrode polarization. Moreover, the gain at $50 / 60 \mathrm{~Hz}$ should be high enough to guarantee the required accuracy in the buffered signal, as indicated in the third equation of (7) where the open-loop gain of the operational amplifier is $G_{O A}$. As it will be clear in the next paragraph, in case of single-ended acquisition, the noise of amplifier OA1 directly contributes to the input referred noise of the signal acquisition chain and needs therefore to be minimized. The component used for the two operational amplifier is Analog Devices AD8629 [22], a dual amplifier having $120 \mathrm{~dB}$ large signal voltage gain, $100 \mathrm{pA}$ input bias current, less than $10 \mathrm{pF}$ input capacitance, $1 \mathrm{~mA}$ quiescent current per amplifier, peak-to-peak noise of $0.5 \mu V_{R M S}$ (integrated from 0.1 and $10 \mathrm{~Hz}$ ) and $22 \mathrm{nV} /$ sqrt $\mathrm{Hz}$ voltage noise density at $1 \mathrm{KHz}$. It should be pointed out that the choice of this operational amplifier is partially due to guarantee maximum reconfigurability in the test system and is not optimal in terms of power consumption in all the different configurations. For differential acquisition with DgRL, both OA1 and OA2 could be low-power amplifiers with no particular restrictions on noise performances. For single-ended acquisition only noise specs for OA2 could be relaxed. Overall power consumption of the DgRL section is summarized in Table I.
TABLE I

BREAKDOWN OF DGRL CIRCUIT POWER CONSUMPTION

\begin{tabular}{|c|c|c|}
\hline Block & Component & Current Consumption \\
\hline \hline References & ADR433 & $560 \mu \mathrm{A}$ \\
\hline Voltage Regulator & ADP3330-3 & $200 \mu \mathrm{A}$ \\
\hline Dual Op.Amp & AD8629 & $2 \mathrm{~mA}$ \\
\hline
\end{tabular}

\section{B. EEG Signal Acquisition}

Acquisition of the EEG signal is performed by means of two Texas Instruments ADS1298, analog front-ends especially designed for low noise acquisition of bio-potentials. Each input is low-pass filtered by a first order passive RC filter with $35 \mathrm{KHz}$ cut-off frequency $(\mathrm{R}=18 \mathrm{~K} \Omega, \mathrm{C}=10 \mathrm{nF})$.

Each device acquires data from 8 electrodes; each channel performs single-ended amplification of the input signal, since the inverting input of each channel is connected to a common reference REF tied to a fixed potential with respect to isolated ground G1. Since the DgRL circuit will force the isolated ground G1 and the REF input of the analog front-end to follow the potential detected on electrode $E_{C M}$, acquired data will implicitly be referenced to that potential. Sample rate is programmed to be $500 \mathrm{SPS}$, for a cut-off frequency of $131 \mathrm{~Hz}$. The input programmable gain amplifier has its gain fixed to 6 .

The overall noise will be given by

$$
\begin{aligned}
& \frac{v_{n, S E}}{\sqrt{\Delta f}}= \\
& =\sqrt{\left(\frac{v_{n, A D C}}{\sqrt{\Delta f}}\right)^{2}+\left(\frac{v_{n, O A 1}}{\sqrt{\Delta f}}\right)^{2}+\left(\frac{v_{n, R E F 2}}{\sqrt{\Delta f}}\right)^{2}+\left(\frac{v_{n, R E F 3}}{\sqrt{\Delta f}}\right)^{2}}
\end{aligned}
$$

where $v_{n, A D C} / \sqrt{\Delta f}$ is the noise spectral density for one channel of the front-end, $v_{n, O A 1} / \sqrt{\Delta f}$ is that of operational amplifier OA1 and $v_{n, R E F 2} / \sqrt{\Delta f}$ and $v_{n, R E F 3} / \sqrt{\Delta f}$ are those of voltage references. As pointed out at the beginning of this section, the presence of REF2 and REF3 is not strictly necessary if bipolar power supply is used. In this case the two corresponding contributions can be removed from (9).

Differential acquisition can be performed in the digital domain by subtracting the signal on one of the 16 channels to that on every other channel. In this case, the overall noise will be

$$
\frac{v_{n, D}}{\sqrt{\Delta f}}=\sqrt{2 \cdot\left(\frac{v_{n, A D C}}{\sqrt{\Delta f}}\right)^{2}} .
$$

In order to minimize coupling of digital noise to the analog ground, the power supplies V1A and V1D are provided by two separate voltage regulators, both delivering a stabilized voltage of $3 \mathrm{~V}$ with respect to $V_{G 1}$. The overall power consumption of the EEG signal acquisition section is summarized in Table II.

\section{Performances and Test Results}

\section{A. Electrical Characterization and CMR Performance Evaluation}

System characterization is performed at first from the electrical standpoint. Comparisons are performed between acquisi- 


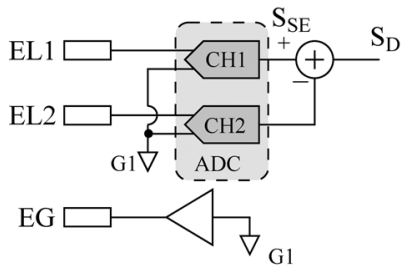

(a)

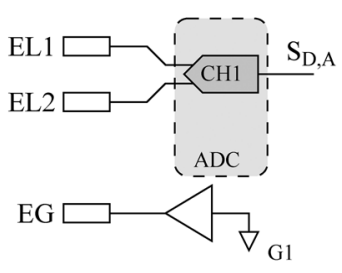

(b)

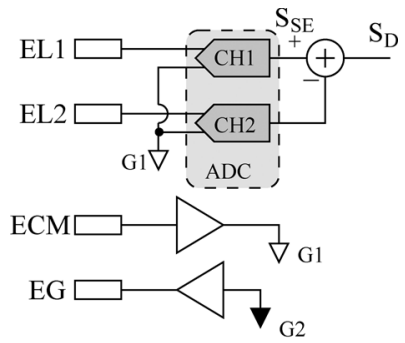

(c)

Fig. 5. Sketches for possible configurations of the acquisition system for testing purposes (considering 2 channels among the 16 available in the system). (a) Single-ended $\left(S_{S E}\right)$ and digital differential $\left(S_{D}\right)$ acquisition without DgRL (the difference is performed in the digital domain after conversion by two separate channels). (b) Analog differential acquisition $\left(S_{D, A}\right)$ without DgRL (a single ADC channel is used, with the differential signal at its inputs). (c) Single-ended $\left(S_{S E}\right)$ and digital differential $\left(S_{D}\right)$ acquisition with DgRL.

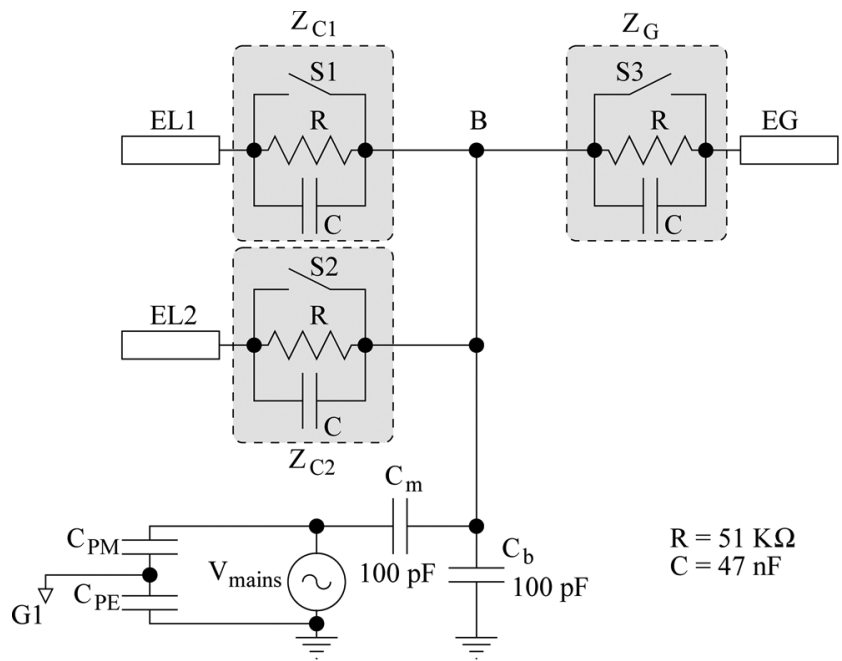

(a)

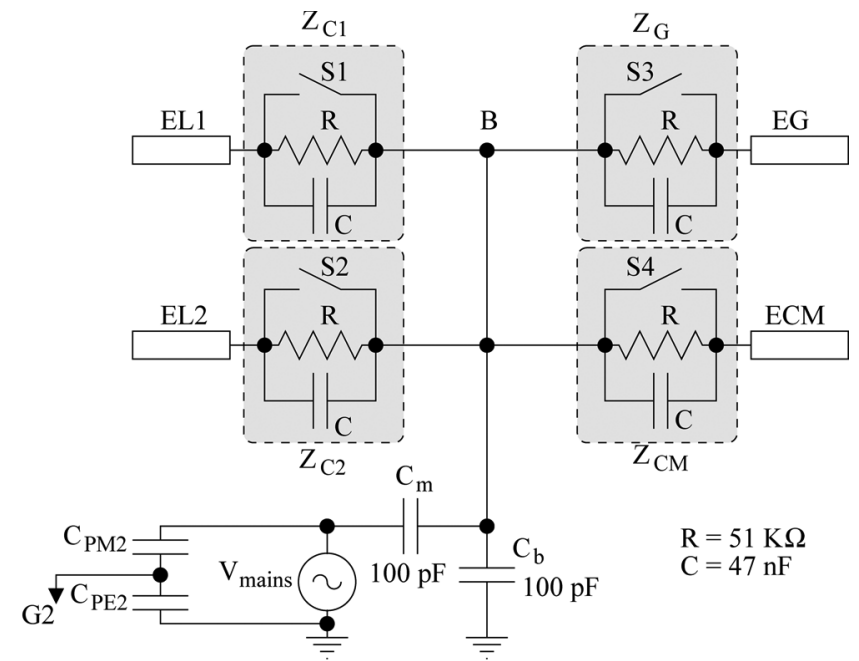

(b)

Fig. 6. Test circuit for CMR as prescribed by IEC60601-2-27 standard for (a) test setup in Fig. 5(a) and (b). (b) Test setup in Fig. 5(c).

TABLE II

BREAKdown of ACQUisition Circuit POWER CONSUMPTION

\begin{tabular}{|c|c|c|}
\hline Block & Component & Current Consumption \\
\hline \hline Front-End + ADC & ADS1298 & $2 \times 3.45 \mathrm{~mA}$ \\
\hline References & ADR443 & $2 \times 3 \mathrm{~mA}$ \\
\hline Voltage Regulators & ADP3330-3 & $2 \times 200 \mu \mathrm{A}$ \\
\hline Digital Isolator & ADUM2401 & $<1 \mathrm{~mA}$ \\
\hline
\end{tabular}

tion of signals with and without the presence of the DgRL circuit for both single-ended and differential measurement.

Fig. 5 shows the test setups which are taken into account for characterization. Fig. 5(a) presents a single-ended acquisition $\left(\mathrm{S}_{S E}\right)$ with the inverting input of each channel of the ADC tied to a fixed voltage. The signals on two electrodes can possibly be subtracted after analog to digital conversion to perform a digital differential acquisition $\left(\mathrm{S}_{D}\right)$. In Fig. 5(b), differential acquisition $\left(\mathrm{S}_{D, A}\right)$ is performed by subtracting the signals directly in the analog domain at the ADC input by connecting the inverting input of the ADC channels to one electrode. These solutions do not adopt DgRL and patient grounding is performed by means of electrode EG connected to a fixed potential through the protection resistor. Fig. 5(c) presents the solutions for testing the system with DgRL. Again, the signals on two electrodes can be subtracted after analog to digital conversion to perform a differential acquisition in the digital domain $\left(\mathrm{S}_{D}\right)$.

The circuits in Fig. 6 are connected to the inputs of the EEG acquisition system to evaluate the CMR performances in the different configurations shown in Fig. 5 as prescribed by standard IEC-60601-2-27 [10]. In particular, the circuit in Fig. 6(a) is used for signal acquisition without DgRL [Fig. 5(a) and (b)], while the circuit in Fig. 6(b) is used when DgRL is present [Fig. 5(c)]. A $20 \mathrm{~V}$ peak-to-peak sine wave is used as the excitation signal $V_{\text {mains }}$. The value of coupling capacitors from the ac main and earth ground are equal to $100 \mathrm{pF}$. Two additional parasitic capacitances $C_{P E}\left(C_{P E 2}\right)$ and $C_{P M}\left(C_{P M 2}\right)$ are considered between the earth and system ground G1 (G2) and between mains and G1 (G2) respectively. The first one is kept fixed at $220 \mathrm{pF}$, while the second one is varied between 0 and $22 \mathrm{pF}$. These values are compatible to those of non-battery operated systems where the interference leaking from mains connection is significantly higher than in portable systems [3]. In (5)-(8), the effect of these parasitic capacitances is taken into account in parameter $\alpha_{1}\left(\alpha_{2}\right)$. Electrodes are modeled using resistor $\mathrm{R}$ and capacitor $\mathrm{C}$ connected in parallel as illustrated. The values of $\mathrm{R}$ and $\mathrm{C}$ are $51 \mathrm{k} \Omega$ and $47 \mathrm{nF}$, respectively. Four switches allow one to short these impedances, so as to simulate a complete mismatch in the signal differential path. 
(a)

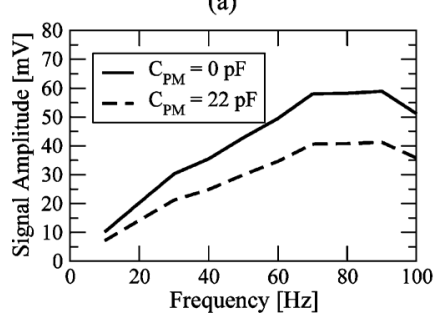

(c)

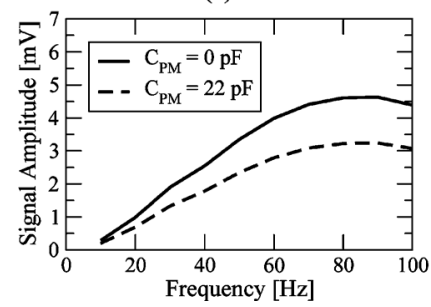

Fig. 7. Common mode signal measured at the output of the different configurations of the acquisition system presented in Fig. 5, with input RC filter and with contact impedance imbalance (one of the switch in Fig. 6 closed). (a) Single Ended - No DgRL. (b) Digital Differential - No DgRL. (c) Analog Differential - No DgRL. (d) Single Ended - with DgRL.

In Fig. 7 we characterize the behavior of the full system with the first order RC input filter and considering a complete mismatch in the signal differential path. IEC-60601 standard prescribes that, for a $50 / 60 \mathrm{~Hz}$ sine wave excitation signal with an amplitude of $20 V_{R M S}$ in the test setup of Fig. 6, the differential output signal should be below $1 \mathrm{mV}$ peak-to-peak, corresponding to a CMR of $95 \mathrm{~dB}$ with respect to the mains signal. Fig. 7(a) and (b) represent respectively the signals at output $\mathrm{S}_{S E}$ and $\mathrm{S}_{D}$ of the circuit in Fig. 5(a). The first graph shows the amplitude of the residual main interference in the system's common mode which is due to the finite impedance between the ground electrode (EG) driver and the common node B. The peak-to-peak amplitude of the interference at 50-60 Hz lies approximately between 25 and $50 \mathrm{mV}$, depending on the value of the parasitic capacitance $C_{P M}$. A higher value for $C_{P M}$ increases the coefficient $\alpha_{1}$ in (7), reducing the effective amplitude of the common mode signal $V_{C M}=V_{B}-V_{G 1}$ at the input of the front-end. The common mode signal increases with increasing frequency since because of the resistive component, the impedance modelling electrode behaviour $\left(Z_{G}\right.$ in (7)) decreases less than the reactance associated to the parasitic capacitances in $(7)$.

Fig. 7(b) shows the signal obtained in computing the difference on the two electrodes after analog to digital conversion. In order to consider a best-case scenario for the analysis of the reference system without DgRL, only channels on the first ADS1298 are considered. In this case, the output signal reduces to approximately $3 \mathrm{mV}$ at $50-60 \mathrm{~Hz}$ and the resulting CMR is approximately $73 \mathrm{~dB}$, more than $20 \mathrm{~dB}$ below the required value.

Fig. 7(c) shows the results of differential measurement, where the two electrode signals are connected to the differential input of the ADS1298 as depicted in Fig. 5(b). The resulting CMR is approximately the same as in Fig. 7(b), due to the fact that the main limitation to CMRR is the mismatch in the impedance between each electrode and the amplifier $\left(C M R R_{Z}\right.$, see (1)
TABLE III

CMR Performances at $50 \mathrm{~Hz}$ of the Presented DGRL With Respect to EXISTING SOLUTIONS

\begin{tabular}{|c||c|c|c|c|c|c|c|}
\hline & {$[14]$} & {$[13]$} & {$[23]$} & {$[19]$} & {$[6]$} & {$[11]$} & DgRL \\
\hline Type & DRL & DRL & DDRL & TDRL & CMFB & CMFF & DgRL \\
\hline $\begin{array}{c}\text { CMR } \\
{[\mathrm{dB}]}\end{array}$ & 40 & 40 & $>70$ & 20 & $<40$ & 25 & $>70$ \\
\hline
\end{tabular}

to (3)) rather than the limited CMRR of the analog front-end $\left(C M R R_{A}\right)$.

Fig. 7(d) presents results when the DgRL circuit is adopted for single-ended acquisition $\left(\mathrm{S}_{S E}\right.$ in Fig. 5). As may be expected from the discussion in Section II, the common mode signal is reduced by a factor approximately equal to $72 \mathrm{~dB}$ with respect to Fig. 7(a) and the dependance on the value of $C_{P M}$ is almost negligible. This results in a CMR in excess of $130 \mathrm{~dB}$. With respect to IFCN requirements for $110 \mathrm{~dB}$ CMRR, a common mode reduction in excess of $70 \mathrm{~dB}$ allows to relax the specs on the CMRR of the acquisition system to less than $40 \mathrm{~dB}$, a value which can be satisfied even in active electrode systems with front-end gain significantly higher than one [5]. This would not be possible if DgRL was substituted by a standard DRL loop, since the common mode reduction would be confined below $40 \mathrm{~dB}$, requiring a CMRR for the acquisition system alone in excess of $70 \mathrm{~dB}$.

Table III presents a comparison in the CMR performances of the presented DgRL with respect to DRL in commercial system [14], DRL in literature (standard [13], transconductance DRL or TDRL [19] and Digital DRL or DDRL [23]), CMFF [11] and CMFB solution [6]. The only solution providing performances similar to DgRL is DDRL. It should be pointed out that, being based on high-Q digital filters, DDRL requires digital processing and additional $\mathrm{A} / \mathrm{D}$ and $\mathrm{D} / \mathrm{A}$ conversion stages. Moreover, it can perform poorly in case of non-stationary interference due to, e.g., home appliances or higher frequency noise (e.g., harmonics of interference from the mains and fluorescent light noise)[19].

\section{B. Functional Characterization}

EEG is acquired after mild treatment of the skin consisting in a brief cleaning of the electrode areas with a dry cloth. The electrodes are standard, passive, syntherized Ag/AgCl cup electrodes with $10 \mathrm{~mm}$ diameter and a 1.5 meter cable; the electrodes are attached to the skin surface by means of conductive adhesive paste. The electrode positions are a subset of those of the 10-20 system. Fig. 8 shows noise measurement in single-ended acquisition, obtained shorting the electrodes by means of EEG conductive paste. The noise component at $50 \mathrm{~Hz}$ is only slightly visible in the signal spectrum. The integrated noise between 0.5 and $100 \mathrm{~Hz}$ is $1.58 \mu \mathrm{V}_{R M S}$, which reduces to $1.03 \mu \mathrm{V}_{R M S}$ if the difference between two channels is computed after analogto-digital conversion (differential acquisition of signal $\mathrm{S}_{D}$ in Fig. 5(c)). From the ADS1298 datasheet, for a sample rate of 500 SPS and a gain of the input amplifier of 6 , the input referred-noise for a single ADC channel is $0.7 \mu \mathrm{V}_{R M S}$ (averaged on at least 1000 samples), which, applying (10), leads to approximately $1 \mu \mathrm{V}_{R M S}$, in line with differential measurement results. 

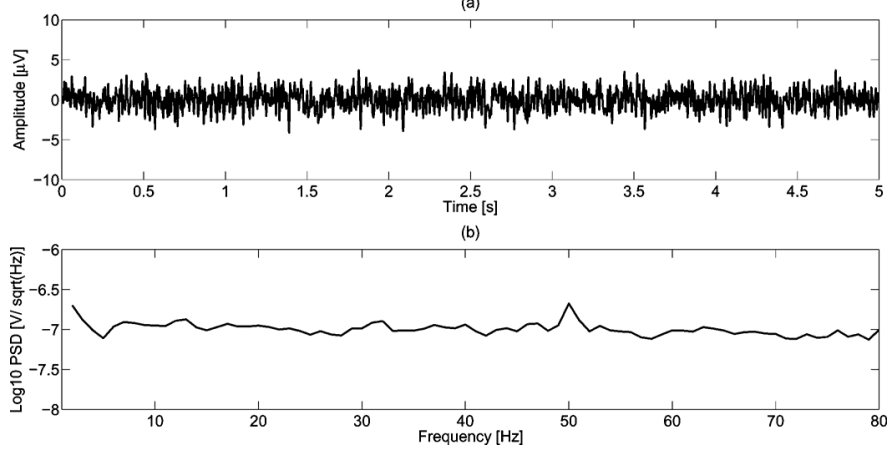

Fig. 8. (a) Noise measurement in the time-domain and (b) noise power spectrum for single-ended acquisition with DgRL.

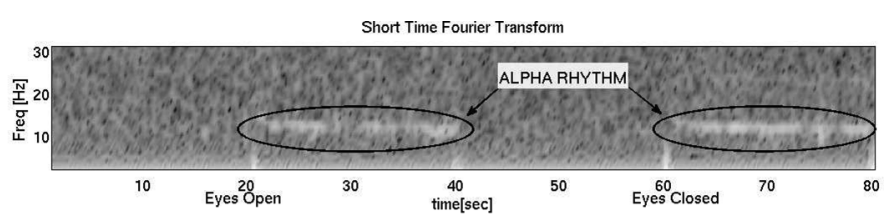

(a)

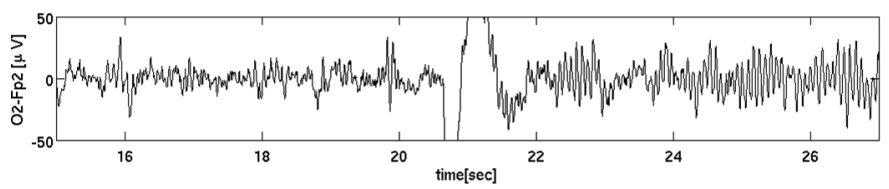

(b)
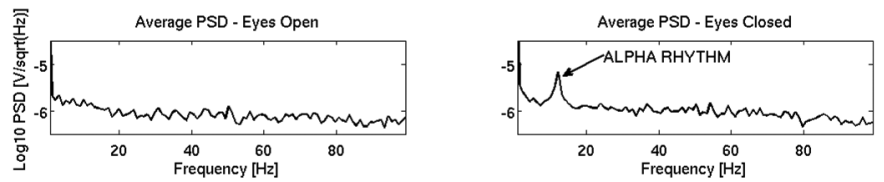

(c)

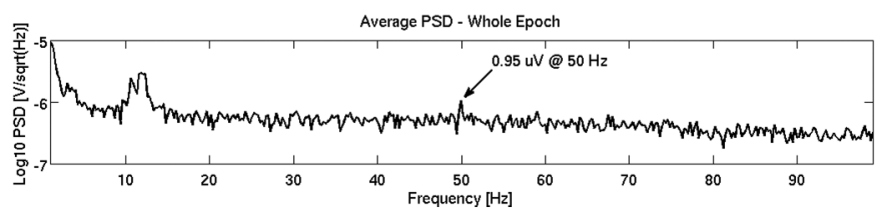

(d)

Fig. 9. EEG signal acquisition in single-ended configuration with DgRL in O2 versus Fp2. (a) Short time fourier tranform showing spectral content increase in the time intervals where the subject has eyes closed ([20-40] and [60-80] seconds). (b) signal in the time-domain during eye transition from open to closed. (c) PSD averaged in Eyes Open and Closed time intervals respectively. (d) PSD averaged on the whole epoch.

For EEG signal acquisition, Fp1 is used as the ground electrode (EG), while Fpz is the one which detects the common mode voltage for the DgRL circuit (ECM). Before proceeding with signal acquisition, we waited 5 minutes to allow for settling of the interface between electrode and skin. The subject was instructed to switch between eyes open and eyes closed every 20 seconds. Fig. 9 shows results from acquisition on electrode $\mathrm{O} 2$ with respect to Fp2, which corresponds to the ECM electrode of the DgRL circuit. The upper part of the graph shows the Short Time Fourier Transform (STFT) of the acquired signal, which is band-pass filtered between 0.5 and $100 \mathrm{~Hz}$ by means of a fourth order Butterworth IIR filter. It can be observed that no common mode interference from the mains $(50 \mathrm{~Hz}$ frequency) is present
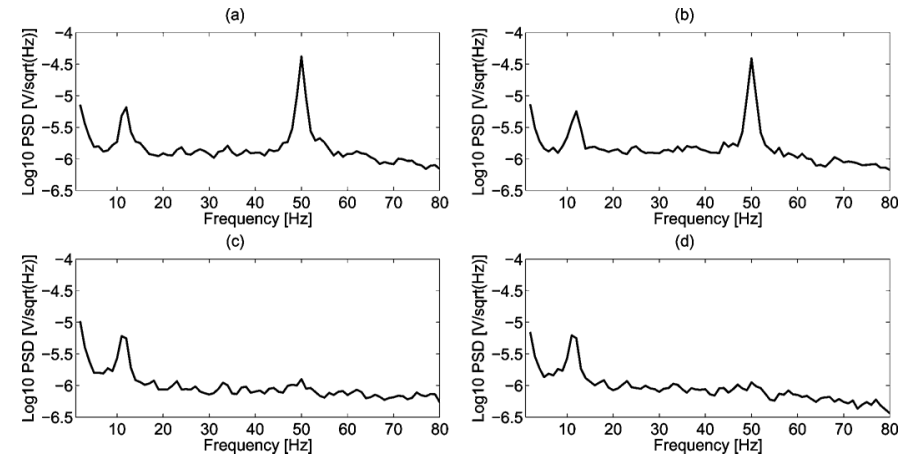

Fig. 10. Comparison in the spectra acquired in $\mathrm{O} 2$ versus Fp2, with the subject keeping his eyes closed, comparing results in case the DgRL is absent or present. The common mode signal on the subject at $50 \mathrm{~Hz}$ is $2.5 \mathrm{mV}_{R M S}$. (a) Digital differential acquisition. (b) Analog differential acquisition. (c) Single ended acquisition with DgRL. (d) Digital differential acquisition with DgRL.

in the acquired signal which clearly shows an alpha rhythm at approximately $12 \mathrm{~Hz}$ frequency when the subject keeps his eyes closed. This is visible in the time domain representation of the acquired signal as well, which is presented in the central part of Fig. 9. In order to improve the graph readability, the acquired signal is band-pass filtered between 0.5 and $40 \mathrm{~Hz}$ by means of a fourth order Butterworth IIR filter. The evident eye movement artifact locates the time instant at which the subject closes his eyes. After this event, the amplitude of the EEG signal increases significantly and the alpha rythmic oscillation can easily be identified. The lower part of the graph shows the Power Spectral Density (PSD) of the signal. In the first line, it is averaged over the 20-seconds epochs in which the subject has his eyes open or closed, respectively. Again, it can be observed that interference from the mains is totally cancelled by the DgRL circuit, while alpha rhythm oscillation is present only in the latter case. In the second line, PSD is averaged on the full 80 seconds epoch. Due to the averaging effect, the level of random components in the EEG spectra is lowered and it is possible to observe the level of the $50 \mathrm{~Hz}$ interference, which is limited to less than 1 $\mu \mathrm{V}$. It should be observed that DgRL electrodes and measuring electrode are in the farthest possible positions and the portion of the interference signal which is not common mode can be considered to be the worst.

Fig. 10 shows a comparison of the spectra acquired with the same setup, with the subject keeping his eyes closed, comparing results in case the DgRL is absent or present. The common mode signal on the subject at $50 \mathrm{~Hz}$ has been measured to be $2.5 \mathrm{mV}_{R M S}$, making a single-ended acquisition impossible. Fig. 10(a) shows the output after digital subtraction of the outputs on electrodes $\mathrm{O} 2$ and $\mathrm{Fp} 2$, without DgRL. A significant interference is present in the output signal at $50 \mathrm{~Hz}\left(40 \mu \mathrm{V}_{R M S}\right)$, notably larger than the tone relating to the alpha rhythm. Similar results are obtained in Fig. 10(b), differentially acquiring signals on electrodes $\mathrm{O} 2$ and Fpz (which is connected to the reference input of the ADS1298). When the DgRL is present (Fig. 10(c) and (d)) single-ended and digital-differential measurements (with electrode ECM in Fpz) provide a significant reduction in the $50 \mathrm{~Hz}$ interference, which almost disappears. This corresponds to a reduction of approximately $30 \mathrm{~dB}$ with respect 
to the differential acquisitions in Fig. 10(a) and (b), while the reduction $\left[K_{C M}\right.$ in (4)] with respect to the original common mode signal of $2.5 \mathrm{mV}_{R M S}$ is approximately $65 \mathrm{~dB}$, only slightly lower than the value estimated in Section II and the one measured on the test setup of Fig. 6. This is especially remarkable if we consider that it enables a single-ended acquisition having substantially the same performances of its differential counterpart.

\section{CONCLUSIONS}

We have presented a novel Driving Right Leg circuit (DgRL) for improving common mode rejection in bio-potential acquisition systems. Unlike standard grounding or DRL circuits which rely on one or more electrodes to fix the patient voltage to a value as stable as possible with respect to the isolated instrumentation ground, the DgRL circuit fixes the instrumentation ground to be stable with respect to the potential on the subject, as detected by one electrode. We have presented an analysis of the improvement in terms of CMR which can be achieved without any risk of incurring in system instability, unlike what happens with standard DRL circuits. The DgRL has been integrated in a 16-channel bio-potential acquisition system in order to verify its performances. Characterization of the system is provided from both the electrical and functional stand-point, showing how DgRL can reduce the common mode signal of a factor in excess of $70 \mathrm{~dB}$ (at least $30 \mathrm{~dB}$ more than a classic DRL loop) and successfully cancel power line interference without any need for differential acquisition and signal post-processing or filtering. EEG signal acquistion on subjects shows how the common mode interference is almost completely cancelled even for single-ended acquisition. This is particularly meaningful as it enables the circuit to be used in systems based on active electrodes, relaxing specifications in terms of gain accuracy and increasing the gain achievable by the first amplification stages which are integrated in the electrodes.

\section{REFERENCES}

[1] M. R. Nuwer et al., "IFCN standards for digital recording of clinical EEG," Electroencephalogr. Clin. Neurophysiol., vol. 106, no. 3, pp. 259-261, Mar. 1998

[2] R. Oostenveld and P. Praamstra, "The five percent electrode system for high-resolution EEG and ERP measurements," Clin. Neurophysiol., vol. 112, no. 4, pp. 713-719, 2001.

[3] A. C. MettingVanRijn, A. Paper, and C. A. Grimbergen, "High quality recording of bioelectric events. Part 1: Interference reduction, theory and practice," Med. Biol. Eng. Comput., no. 28, pp. 389-397, Sep. 1990.

[4] M. Guermandi, R. Cardu, E. Franchi, and R. Guerrieri, “Active electrode IC combining EEG, electrical impedance tomography, continuous contact impedance measurement and power supply on a single wire," in Proc. ESSCIRC, Sep. 2011, pp. 335-338.

[5] M. Guermandi, R. Cardu, E. Franchi, and R. Guerrieri, "Active electrode IC for EEG and electrical impedance tomography with continuous monitoring of contact impedance," IEEE Trans. Biomed. Circuits Syst., vol. 9, no. 1, pp. 21-33, Feb. 2015.

[6] J. Xu et al., "A $160 \mu \mathrm{W}$ 8-Channel active electrode system for EEG monitoring," IEEE Transt. Biomed. Circuits Syst., vol. 5, no. 6, pp. 555-567, Dec. 2011.

[7] Y. M. Chi and G. Cauwenberghs, "Micropower non-contact EEG electrode with active common-mode noise suppression and input capacitance cancellation," in Proc. 31st Annu. Int. Conf. IEEE EMBS, Minneapolis, MN, USA, 2009, pp. 4218-4221.
[8] Y. M. Chi, T.-P. Jung, and G. Cauwenberghs, "Dry-contact and noncontact biopotential electrodes: Methodological review," IEEE Rev. Biomed. Eng., vol. 3, pp. 106-119, 2010.

[9] IEC 60601-1, Medical Electrical Equipment - Part 1: General Requirements for basic Safety and Essential Performance, 3rd ed.

[10] IEC 60601-2-27, Medical Electrical Equipment - Part 2-27: Particular Requirements for the basic Safety and Essential Performance of Electrocardiographic Monitoring Equipment, 3rd ed.

[11] J. Xu et al., "A wearable 8-Channel active-electrode EEG/ETI acquisition system for body area networks," IEEE J. Solid-State Circuits, vol. 49, no. 9, pp. 2005-2016, Sep. 2014.

[12] B. B. Winter and J. G. Webster, "Driven-right-leg circuit design," IEEE Trans. Biomed. Eng., vol. 30, no. 1, pp. 62-66, Jan. 1983.

[13] T. Degen and H. Jackel, "A pseudodifferential amplifier for bioelectric events with DC-offset compensation using two-wired amplifying electrodes," IEEE Trans. Biomed. Eng., vol. 53, no. 2, pp. 300-310, Feb. 2006.

[14] BioSemi, ActiveTwo User Manual, Version 3.2, Jul. 3, 2007.

[15] T. C. Ferree, P. Luu, G. S. Russell, and D. M. Tucker, "Scalp electrode impedance, infection risk, and EEG data quality," Clin. Neurophysiol., vol. 112 , no. 3, pp. 536-544, Mar. 2011.

[16] A. Miller, "Coupling circuit with driven guard," U.S. Patent 4191 195, 1978.

[17] "Low-power, 8-channel, 24-bit analog front-end for biopotential measurements," datasheet [Online]. Available: http://www.ti.com/lit/ ds/symlink/ads1298.pdf, retrieved online May 2015.

[18] V. Acharya, Application Report SBAA188 Improving Common-Mode Rejection Using the Right-Leg Drive Amplifier, Jul. 2011 [Online] Available: http://www.ti.com/lit/an/sbaa188/sbaa188.pdf, retrieved online May 2015

[19] E. M. Spinelli, N. H. Martinez, and M. A. Mayosky, "A transconductance driven-right-leg circuit," IEEE Trans. Biomed. Eng., vol. 46, no. 12, pp. 1466-1470, Dec. 1999.

[20] Quad-Channel Digital Isolators ADuM2400/ADuM2401/ADuM2402, datasheet [Online]. Available: http://www.ftdichip.com/Support/Documents/DataSheets/Modules/DS FT2232H_Mini_Module.pdf, retrieved on-line May 2015

[21] FT2232H Mini Module - USB Hi-Speed FT2232H Evaluation Module, datasheet [Online]. Available: http://www.ftdichip.com/Support/Documents/DataSheets/Modules/DS FT2232H Mini_Module. pdf, retrieved on-line May 2015

[22] Zero-Drift, Single-Supply, Rail-to-Rail Input/Output Operational Amplifier, datasheet [Online]. Available: http://www.analog.com/ static/imported-files/data_sheets/AD8628_8629_8630.pdf, retrieved on-line Oct. 2014

[23] M. A. Haberman and E. M. Spinelli, "A multichannel EEG acquisition scheme based on single ended amplifiers and digital DRL," IEEE Trans. Biomed. Circuits Syst., vol. 6, no. 6, pp. 614-618, Dec. 2012.

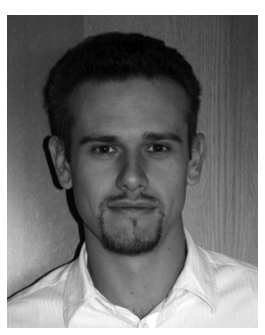

Marco Guermandi received the M.Sc. and Ph.D. degrees from the University of Bologna, Bologna, Italy, in 2005 and 2009, respectively.

Since 2009, he has been with the Advanced Research Center on Electronic Systems "E. De Castro," University of Bologna. His current research interests include the design of high-performance application specific integrated circuits for biomedical applications and the development of biomedical instrumentation for neural imaging from bioelectrical data, both from hardware and algorithm standpoint.

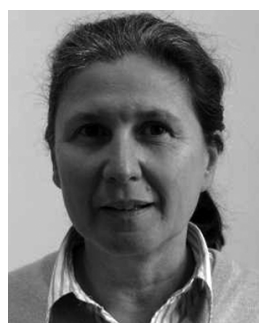

Eleonora Franchi Scarselli (M'98) received the M.S. degree in electrical engineering and the Ph.D. degree in electrical engineering and computer science from the University of Bologna, Bologna, Italy.

From 1994 to 2004, she was a Research Assistant with the Faculty of Engineering, University of Bologna, where she has been an Associate Professor since 2005 and currently teaches design of digital integrated circuits. She is also with the Advanced Research Center on Electronic Systems (ARCES) at the University of Bologna, where she has been and is currently involved in research activities concerning design of circuits and system for RF and sensor applications, and for enabling wireless 3-D communication. 


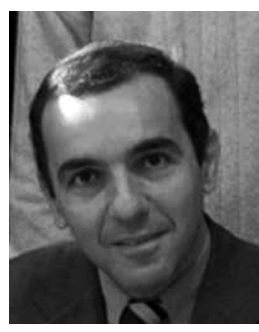

Roberto Guerrieri received the Dr.Eng. and Ph.D. degrees from the University of Bologna, Bologna, Italy, in 1980 and 1986 respectively.

After working in the Department of Electrical Engineering and Computer Sciences, University of California, Berkeley, Berkeley, CA, USA, as a Visiting Researcher and at the Massachusetts Institute of Technology, Cambridge, MA, USA, as a Visiting Scientist, he joined the University of Bologna, where he is a Full Professor and teaches design of integrated systems. His research interests

are in various aspects of integrated circuit modeling and design, including digital systems and biometric sensors, and applications of microelectronics to biotechnology. His work on VLSI design has been cited by widely-read magazines, such as Nikkei and Electronic Design, and documented in more than 90 scientific papers. He has founded two start-up companies in the field of biomedical devices for patient monitoring in cancer therapy.

Dr. Guerrieri won the best paper award of the IEEE TRANSACTIONS ON SEMiconductor MANUfACtURING in 1992 for his research carried out on issues related to the modeling of various IC manufacturing steps. In 2004, he received an ISSCC best paper award for his work in the area of silicon-based lab-on-a-chip. 